\title{
Paradigma atual da comunicação científica e introdução da revista Pesquisa Agropecuária Brasileira (PAB) no canal eletrônico
}

Patrícia Rocha B. Bertin

Graduação em Ciências Biológicas pela

Universidade de Brasília (1999) e mestrado em Patologia Molecular pela Universidade de Brasília (2002); Pesquisadora B da Empresa Brasileira de Pesquisa Agropecuária.

Juliana Meireles Fortaleza

Graduação em Engenharia Agronômica pela Universidade de Brasília (1999) e mestrado em Ciências Agrárias pela Universidade de Brasília (2002); Pesquisadora B da Empresa Brasileira de Pesquisa Agropecuária.

Allert Rosa Suhet

Graduação em Engenharia Agronômica pela Universidade Federal Rural do Rio de Janeiro (1971) e mestrado em Solos e Nutrição de Plantas pela Escola Superior de Agricultura Luiz de Queiroz (1975); Pesquisador B da Empresa Brasileira de Pesquisa Agropecuária.

Este trabalho tem por objetivo discutir a evolução da comunicação científica formal desde o surgimento da Internet, posicionar a revista Pesquisa Agropecuária Brasileira ( $P A B)$ nesse contexto e analisar o impacto da introdução da $P A B$ no meio eletrônico. Para isto, analisouse a evolução das citações recebidas pela revista PAB no período de 1997 a 2006, a partir de dados obtidos na biblioteca eletrônica SciELO. Entre 2001 e 2006, o acesso à página da PAB eletrônica na SciELO quadruplicou, enquanto os acessos diretos aos artigos aumentaram 50 vezes. Nesse mesmo período, o número de citações recebidas duplicou, chegando a 934 citações recebidas por periódicos participantes da SciELO em 2006. Paralelamente, o número de manuscritos recebidos aumentou no período analisado. Considerados em conjunto, esses dados indicam um progresso rumo à legitimação do periódico eletrônico para a disseminação da informação pela comunidade científica. 
Palavras-chave: Comunicação científica; Pesquisa Agropecuária Brasileira; Periódico científico; Publicação eletrônica; Acesso livre; Fator de Leitura.

\title{
Current scenario of scientific communication and the introduction of the Brazilian Journal of Agricultural Research (PAB) in the electronic media
}

\begin{abstract}
This work aims to discuss the evolution of scientific formal communication since Internet emergence, to place the Brazilian Journal of Agricultural Research - PAB - into this international context, and to analyze the impact of introducing this periodical into the electronic media. The number of citations received by the $P A B$ from 1997 to 2006 was evaluated, through data obtained from SciELO electronic library. From 2001 to 2006, the number of accesses to PAB's homepage increased four times, whilst the direct accesses to PAB's articles augmented 50 times and the number of citations received duplicated, and reached the number of 934 in the last year. Additionally, the number of manuscripts received by the journal, for evaluation, increased. Taken together, these data demonstrate a progress through the legitimation of the electronic journal for information dissemination, by scientific community.
\end{abstract}

Keywords: Scientific communication; Pesquisa Agropecuária Brasileira; Scientific periodical; Electronic publishing; Open access; Reading Factor.

Recebido em 11.05.2007 Aceito em 01.10.2007

\section{Introdução}

Tópico muito explorado e discutido pela Ciência da Informação, a comunicação científica é definida como a troca de informações entre membros da comunidade científica, desde o momento em que o cientista concebe uma idéia para pesquisar, até o momento em que os resultados da pesquisa sejam aceitos como constituintes do conhecimento científico. Ou seja, estão inseridas, no contexto da comunicação científica, atividades associadas à produção, à disseminação e ao uso da informação. GARVEY (1979, p. 10) define comunicação científica como sendo: 
[...] o campo de estudo do espectro total de atividades informacionais que ocorrem entre os produtores da informação científica, desde o momento em que eles iniciam suas pesquisas até a publicação de seus resultados e sua aceitação e integração a um corpo de conhecimento científico.

Entre as variadas formas, o periódico científico consiste no principal canal formal de disseminação da ciência e assume, com destaque, as funções de 'certificação' (controle de qualidade), de arquivo ou memória científica, e de registro de autoria da descoberta científica (MUELLER, 1999).

No meio científico, o prestígio dos periódicos é determinado por um sistema de avaliação baseado em vários indicadores, como: quantidade de artigos publicados, índice de citação e visibilidade internacional. As principais bases que fazem o levantamento desses dados são o Institute for Scientific Information (ISI), nos Estados Unidos, e a Scientific Electronic Library Online (SciELO), no Brasil.

Assim como a comunicação informal entre autores e leitores e a colaboração entre pesquisadores foram facilitadas por meio dos correios eletrônicos (MUELLER; PASSOS, 2000), a rápida evolução das tecnologias de informação, nas últimas décadas, alimentou 0 debate sobre a dinamização do sistema de publicação científica vigente, pela utilização da mídia eletrônica na comunicação científica. Surgiram os primeiros periódicos exclusivamente disponíveis na World Wide Web, pioneiros e inovadores, enquanto revistas tradicionais passaram a adotar versões eletrônicas de seus conteúdos.

\section{Um pouco sobre a Pesquisa Agropecuária Brasileira (PAB)}

Atualmente em seu $42^{\circ}$ ano de publicação ininterrupta, a PAB tem contribuído com a divulgação de informações científicas inéditas, provenientes de pesquisas realizadas em instituições brasileiras e estrangeiras, relacionadas às diversas especialidades das ciências agrárias. Ao mesmo tempo em que faz o registro documental, a PAB atesta o esforço de inúmeros cientistas na busca de avanço do conhecimento científico - a base para o desenvolvimento de tecnologias inovadoras e para o progresso da agropecuária brasileira. Desde 1982 a revista é publicada mensalmente pela Empresa Brasileira de Pesquisa Agropecuária.

A indexação pelos principais índices internacionais de revistas científicas das ciências agrícolas - ISI (Web of Science e Current Contents: Agriculture, Biology \& Environmental Science) e CAB Abstracts e AGRIS - e a participação na biblioteca de revistas científicas SciELO (Scientific Electronic Library Online) atestam o reconhecimento da comunidade científica quanto à qualidade, relevância e contribuição da revista como instrumento legítimo de documentação da informação científica e como agente do desenvolvimento científico. 
A classificação dos veículos utilizados pelos programas de pósgraduação para a divulgação da produção intelectual de seus docentes e alunos, promovida pela Capes, tem revelado bom índice de desempenho da revista, conforme consta em sua lista Qualis - com conteúdo multidisciplinar, a PAB está enquadrada na categoria de qualidade " $A$ " em dez das dezessete áreas de atuação em que é avaliada, nos âmbitos nacional e internacional.

A revista Pesquisa Agropecuária Brasileira adota o procedimento de revisão cega (blind review), em que os avaliadores realizam sua análise e procedem ao julgamento sem conhecimento da autoria dos originais. Segundo o modelo adotado, os autores também não têm conhecimento do nome dos avaliadores de seus originais. Desta forma, o anonimato é bidirecional.

O presente trabalho tem por objetivo discutir a evolução na comunicação científica formal desde o surgimento da Internet, posicionar a revista Pesquisa Agropecuária Brasileira (PAB) neste contexto, e analisar o impacto da introdução da PAB no meio eletrônico.

\section{Metodologia}

A primeira fase desse trabalho consistiu em uma revisão teórica da evolução da comunicação científica formal ao longo dos últimos anos, considerando o advento da Internet e o movimento de Acesso Livre à informação científica. Buscou-se, ainda, recuperar parte da história dos 42 anos da revista PAB e posicionar a revista no contexto mundial de comunicação científica.

\subsection{A avaliação de impacto por meio do fator de leitura}

$\mathrm{Na}$ literatura científica, um critério de reconhecimento de valor de um artigo é o grau de influência que exerce sobre artigos secundários. A citação por outros artigos é aceita como uma estimativa da importância de uma publicação. No presente trabalho, utilizou-se como índice bibliométrico o fator de leitura (reading factor) para análise de impacto da revista PAB, segundo metodologia proposta por Darmoni et al. (2002). Especificamente, o fator de leitura contabiliza o número de vezes que determinada publicação eletrônica é visitada, tendo correlação direta com o fator de impacto de uma revista.

Os dados de acessos à página da revista e de acessos diretos a seus artigos foram obtidos dos servidores da SciELO (Scientific Electronic Library Online, http://www.scielo.br), uma biblioteca eletrônica que abrange uma coleção selecionada de periódicos científicos brasileiros e objetiva o desenvolvimento de uma metodologia comum para a preparação, o armazenamento, a disseminação e a avaliação da produção científica em formato eletrônico. Os 176 periódicos atualmente depositados na plataforma SciELO são regularmente avaliados segundo requisitos de qualidade que determinam sua permanência na coleção eletrônica e que são também aplicados aos periódicos que pretendem 
integrar-se a ela (SCIENTIFIC ELECTRONIC LIBRARY ONLINE - SCIELO, 2007).

Como unidade de análise, foram tomadas as citações recebidas por artigos publicados na PAB no período de 1997 a 2006, nas diversas seções da revista. O universo totaliza 2.220 artigos publicados. Foram consideradas, para esse trabalho, as citações recebidas pela PAB dos 176 títulos credenciados na SciELO, como periódicos citantes.

Para avaliar os impactos causados à revista PAB por sua introdução na biblioteca eletrônica de periódicos da SciELO foram, portanto, utilizados dados estatísticos fornecidos pela biblioteca. Os dados analisados foram: número de citações de artigos da PAB por artigos secundários; total de acessos registrados aos artigos da PAB através da plataforma Scielo; total de acessos diretos aos artigos da PAB; e a relação entre a quantidade de artigos publicados pela PAB e o total de acessos aos seus artigos.

\section{A adoção da mídia eletrônica por periódicos científicos}

No final da década de 1990, o aumento dos preços das revistas provocou a chamada 'crise dos periódicos', que resultou em lucros menores para os editores e em custos maiores para os indivíduos, bibliotecas, mantenedores de bibliotecas e seus usuários (TENOPIR; KING, 1988). Concomitantemente, o desenvolvimento das novas tecnologias de informação e comunicação, principalmente a Internet, proporcionou à comunicação científica mudanças significativas.

Beneficiando-se da rede internacional de computadores, os periódicos científicos iniciaram um movimento de adoção da mídia eletrônica para publicação. Dentre as vantagens que o periódico eletrônico apresenta, destacam-se: i) o baixo custo de investimento e de produção; ii) não há custos de reprodução e transporte; iii) a possibilidade de utilização de recursos de multimídia (áudio, vídeo, hyperlinks); iv) a redução dos atrasos de publicação; v) a submissão eletrônica de manuscritos; vi) o baixo custo de acesso; vii) a disponibilidade instantânea e global da informação; viii) a facilidade de busca pelos sistemas de busca, que proporciona maior acesso e maior número de citações; e ix) a facilidade de cópia e impressão.

Em razão das vantagens expostas, o modelo de publicação eletrônica favorece o acesso livre à informação científica, impulsionado pela reação de pesquisadores, em todo o mundo, ao modelo tradicional de publicação científica, ao valor exorbitante da assinatura das revistas de maior prestígio no meio científico e ao alto índice de rejeição. 0 movimento de apoio ao acesso livre à informação científica teve início em dezembro de 2001, em Budapeste, em uma reunião promovida pelo Open Society Institute (OSI). A Declaração de Budapeste foi o primeiro documento a definir princípios e estratégias para se concretizar e assegurar o acesso livre à informação científica.

Desde a reunião histórica de Budapeste, cientistas, editores, bibliotecários e demais profissionais da área têm fomentado a discussão, e 
diversos documentos de aprovação ao movimento de apoio ao acesso livre foram divulgados, entre eles a Declaração de Bethesda e a Declaração de Berlim, lançadas em 2003 (SUBER, 2007), e o Manifesto Brasileiro de Apoio ao Acesso Livre à Informação Científica, publicado em 2005 (INSTITUTO BRASILEIRO DE INFORMAÇÃO EM CIÊNCIA E TECNOLOGIA IBICT, 2005). A definição de acesso livre contida na Declaração de Bethesda foi adotada em documentos posteriores e determina a exigibilidade dos seguintes critérios, para que uma publicação possa ser considerada de acesso livre:

1. $\mathrm{O}(\mathrm{s})$ autor(es) e o(s) detentor(es) de direitos de reprodução (copyright) concede $(\mathrm{m})$ a todos os usuários o acesso livre, irrevogável, mundial e perpétuo ao trabalho, assim como uma licença de cópia, uso, distribuição, transmissão e exibição pública, e ainda de produzir e distribuir trabalhos dele derivados, em qualquer meio digital, para qualquer finalidade responsável, condicionado à devida atribuição de autoria; e concedem adicionalmente o direito de produção de uma pequena quantidade de cópias impressas para seu uso pessoal.

2. Uma versão integral do trabalho e de todo o material suplementar, incluindo uma cópia da permissão em um formato eletrônico adequadamente padronizado, é depositada imediatamente após a publicação inicial em um repositório online mantido por uma instituição acadêmica, por uma associação científica, por uma agência governamental ou por qualquer outra organização solidamente estabelecida, que vise a propiciar o acesso livre, a distribuição irrestrita, a interoperabilidade e o arquivamento de longo prazo (para as ciências biomédicas, a PubMed Central se constitui em um repositório desta natureza). (BETHESDA..., 2003).

Utilizando-se da mídia eletrônica, os editores de periódicos têm assumido posicionamentos diversificados: i) adotam política de acesso aberto e disponibilizam o conteúdo gratuitamente na Internet (especialmente comum em periódicos editados por instituições públicas); ii) oferecem versões impressa e eletrônica, mas cobram pelo acesso; iii) disponibilizam os arquivos eletrônicos por alguns meses após a publicação da revista impressa; iv) cobram, dos autores, taxa para sustentar a publicação em acesso aberto; v) permitem acesso aberto a alguns artigos, por fascículo; vi) possibilitam acesso livre por usuários de países com renda per capita baixa; vii) oferecem acesso irrestrito apenas a resumos e ao sumário; viii) sustentados por instituições públicas, oferecem acesso completamente aberto. 


\section{A introdução da PAB na mídia eletrônica}

Em julho de 1997, somando ao movimento constatado de migração de muitos periódicos para a mídia eletrônica e em consonância com o que posteriormente consagrou-se como movimento de acesso livre à informação científica, a PAB publicou seu primeiro fascículo na Internet (http://www.sct.embrapa.br/pab/), com acesso irrestrito, em página hospedada nos servidores da Embrapa Informação Tecnológica. Desde então a revista tem assumido um padrão híbrido de publicação - impressa e eletrônica -, com conteúdos idênticos. Em 2000 a revista foi incorporada ao Projeto SciELO, no endereço http://www.scielo.br/scielo.php?pid=0100-204X\&script=sci_serial/. Desta forma, atualmente os conteúdos da revista podem ser acessados nas páginas da PAB hospedadas no servidor da Embrapa e na plataforma SCiELO.

Desde o lançamento da PAB eletrônica, esforços de digitalização têm sido empenhados, objetivando a publicação retroativa dos fascículos da revista. Atualmente encontram-se integralmente acessíveis ao público, na Internet, os artigos publicados a partir de janeiro de 1991.

Segundo Campos (2003), a própria inserção de um periódico nas bases da SciELO representa uma indicação de qualidade da revista, e pode ser entendida como um índice bibliométrico. Além disso, o número de citações de um artigo indica a influência ou impacto que seu conteúdo produz na comunidade científica da área. Assim, os dados de acesso e de citações da PAB por revistas integrantes da biblioteca eletrônica SciELO são importantes índices do impacto dessa revista.

Com 97 fascículos disponíveis na SciELO, a PAB é a revista com o maior número de fascículos publicados, dentro do grupamento dos 25 periódicos das Ciências Agrárias inscritos na biblioteca eletrônica. No grupamento geral, em número de fascículos disponibilizados na biblioteca eletrônica, a PAB está atrás dos seguintes periódicos: Revista de Saúde Pública, Arquivos Brasileiros de Cardiologia, Cadernos de Saúde Pública, Brazilian Journal of Medical and Biological Research, e Revista do Instituto de Medicina Tropical de São Paulo, que apresentam, respectivamente, 220, 149, 133, 125 e 105 fascículos publicados e estão agrupadas nas áreas de Ciências Biológicas ou Ciências da Saúde, conhecidas como as de maior tradição na comunicação científica.

Como comprovação do interesse da comunidade científica nesse veículo de comunicação, em 2004 a PAB recebeu 736 artigos para avaliação (média de 61 artigos submetidos por mês), número 4 vezes superior à capacidade de publicação da revista naquele ano (180 artigos). Nos anos de 2005 e 2006 houve um aumento médio de $25 \%$ no número de manuscritos recebidos, totalizando 931 e 915 artigos, respectivamente. 


\section{Acesso à PAB eletrônica e análise de impacto segundo método do fator de leitura}

A avaliação dos dados de acesso ao conteúdo da PAB, na plataforma SciELO, revela um número crescente de visitas à página da revista e de acessos diretos aos artigos, desde 2001 (Tabela 1). O número de acessos à página da PAB quadruplicou de 2001 a 2006, o que representa um grande aumento da visibilidade da revista. Por sua vez, a contagem dos acessos diretos aos artigos da PAB, por meio das ferramentas de busca na Internet, aumentou 50 vezes nesse período (Tab. 1).

TABELA 1 -Acessos registrados na página inicial da revista Pesquisa Agropecuária Brasileira na biblioteca eletrônica SciELO e acessos diretos a artigos depositados, de 2001 a 2006

\begin{tabular}{c|c|c|c}
\hline \multirow{2}{*}{ Ano } & \multicolumn{2}{|c|}{ Acessos registrados (no) } & \multirow{2}{*}{$\begin{array}{c}\text { Total de artigos } \\
\text { publicados }\end{array}$} \\
\cline { 2 - 3 } & À página inicial da PAB & A artigos & 195 \\
2001 & 7.409 & 10.060 & 235 \\
2002 & 24.799 & 31.732 & 190 \\
2003 & 23.485 & 45.670 & 180 \\
2004 & 22.101 & 163.817 & 180 \\
2006 & 26.747 & 282.085 & 220 \\
\hline
\end{tabular}

FONTE - Dados extraídos de SciELO (2007).

Esses dados confirmam as proposições de Meadows (1997) sobre a implantação de inovações. De acordo com o autor, a aceitação de inovações normalmente segue a curva em " $S$ ". Inicialmente há um pequeno progresso (a parte inferior do $S$ ), depois as pessoas começam a conhecer o produto e o progresso quanto à aceitação se acelera (a parte do meio do S), e, finalmente, quando a maioria da comunidade aceita a inovação, a taxa de crescimento diminui até que seja atingido o objetivo (a parte superior do $\mathrm{S}$ ).

Considerando os dados da Tabela 1, pode-se afirmar que a revista PAB encontra-se, atualmente, na parte intermediária do $S$; ou seja, o público já conhece a revista e o acesso a seus artigos tem aumentado rapidamente a cada ano. Particularmente entre os anos de 2003 e 2004 os acessos a artigos da PAB na plataforma SciELO aumentaram em $258 \%$ e, desde então, esse número vem aumentando $76,5 \%$ ao ano, apesar de - número de artigos publicados por ano ter experimentado pouca alteração, com uma média de 200 artigos. Isto reflete a legitimação da mídia eletrônica para a disseminação da informação por parte da comunidade científica. As discussões em torno do movimento de acesso livre à informação científica, que tiveram início oficial em 2001 com a declaração de Budapest, a inserção digital e a validação dos periódicos eletrônicos, por meio de processos de avaliação da qualidade, têm 
promovido maior credibilidade às informações científicas divulgadas em meio eletrônico, fazendo com que os usuários da rede tenham maior confiança nos dados ali publicados.

A diferença entre a quantidade de artigos acessados a partir da página inicial da PAB na plataforma SciELO e a quantidade de acessos diretos a artigos depositados deve-se à evolução dos mecanismos de busca na Internet, como o Google e Yahoo, entre outros.

\subsection{Evolução das citações recebidas pela revista}

Muito comumente a análise padrão de um gráfico de citações revela uma curva crescente. Isto porque os cientistas depositam maior confiança em trabalhos desenvolvidos recentemente que já tenham sido validados pela comunidade científica. Existe, assim, um tempo de latência para que a comunidade assimile uma inovação em suas áreas de atuação. Desse modo, artigos mais antigos e validados são cada vez mais citados e, somadas as citações das citações, acaba-se por construir uma realidade em que um artigo é tanto mais citado quanto mais antiga for sua data de publicação. Entretanto, o que se observa nos registros das citações recebidas pela $\mathrm{PAB}$ de periódicos integrantes do projeto SciELO, entre 2001 e 2006, é bastante diferente (Tab. 2).

TABELA 2 - Citações de artigos publicados pela Pesquisa Agropecuária Brasileira concedidas por outros periódicos integrantes da biblioteca SciELO a partir de 1996

\begin{tabular}{cc}
\hline Ano-base & $\begin{array}{c}\text { Citações } \\
\text { recebidas }\end{array}$ \\
\hline 2001 & 547 \\
2002 & 655 \\
2003 & 738 \\
2004 & 874 \\
2005 & 794 \\
2006 & 934 \\
\hline
\end{tabular}

FONTE - Dados extraídos de SciELO (2007).

Nota-se claramente o incremento no número de citações recebidas por ano, de 2001 a 2006. O total de citações recebidas quase duplicou nesse período. Nesse mesmo período o número de assinaturas da revista impressa decresceu. Assim, é possível concluir que a introdução e a publicação ininterrupta de novos fascículos da PAB na mídia eletrônica têm ocasionado o aumento da visibilidade da revista e, conseqüentemente, do seu impacto.

Entre as revistas integrantes da biblioteca SciELO, as que mais citaram a PAB, entre 2001 e 2006, foram a Revista Brasileira de Zootecnia, a Ciência Rural e a Revista Brasileira de Fruticultura, com 533, 464 e 316 citações concedidas à revista, respectivamente (Tab. 3). 
TABELA 3 -Revistas eletrônicas integrantes da biblioteca SciELO que mais citaram artigos da PAB, entre 2001 e 2006(1).

\begin{tabular}{lc}
\hline \multicolumn{1}{c}{ Título do periódico } & Citações de artigos da \\
& PAB \\
\hline Revista Brasileira de Zootecnia & 533 \\
Ciência Rural & 464 \\
Revista Brasileira de Fruticultura & 316 \\
Scientia Agrícola & 266 \\
Revista Brasileira de Ciência do Solo & 260 \\
Bragantia & 214 \\
Horticultura Brasileira & 185 \\
Revista Brasileira de Engenharia Agrícola e Ambiental & 179 \\
Neotropical Entomology & 142 \\
Revista Brasileira de Sementes & 124 \\
\hline
\end{tabular}

FONTE - Dados extraídos de SciELO (2007).

Ao proceder a uma análise mais aprofundada dos registros das citações de artigos da PAB publicados entre 1997 e 2005 por periódicos integrantes do projeto SciELO, observa-se que apenas no ano de 2006 houve $50 \%$ de aumento nas citações de artigos publicados pela PAB entre 1999 e 2000 (Figura 1). Pode-se explicar esse fato pelo incremento no número de artigos publicados por fascículo da revista nos anos de 1999 e 2000. A PAB publicou uma média de 25 artigos por mês nesses dois anos, o que impulsionou positivamente a revista, e pode ser atestado pelo fato de o número de citações manter-se elevado no ano seguinte, apesar de o número de artigos publicados mensalmente ter-se reduzido para 16, em média. 


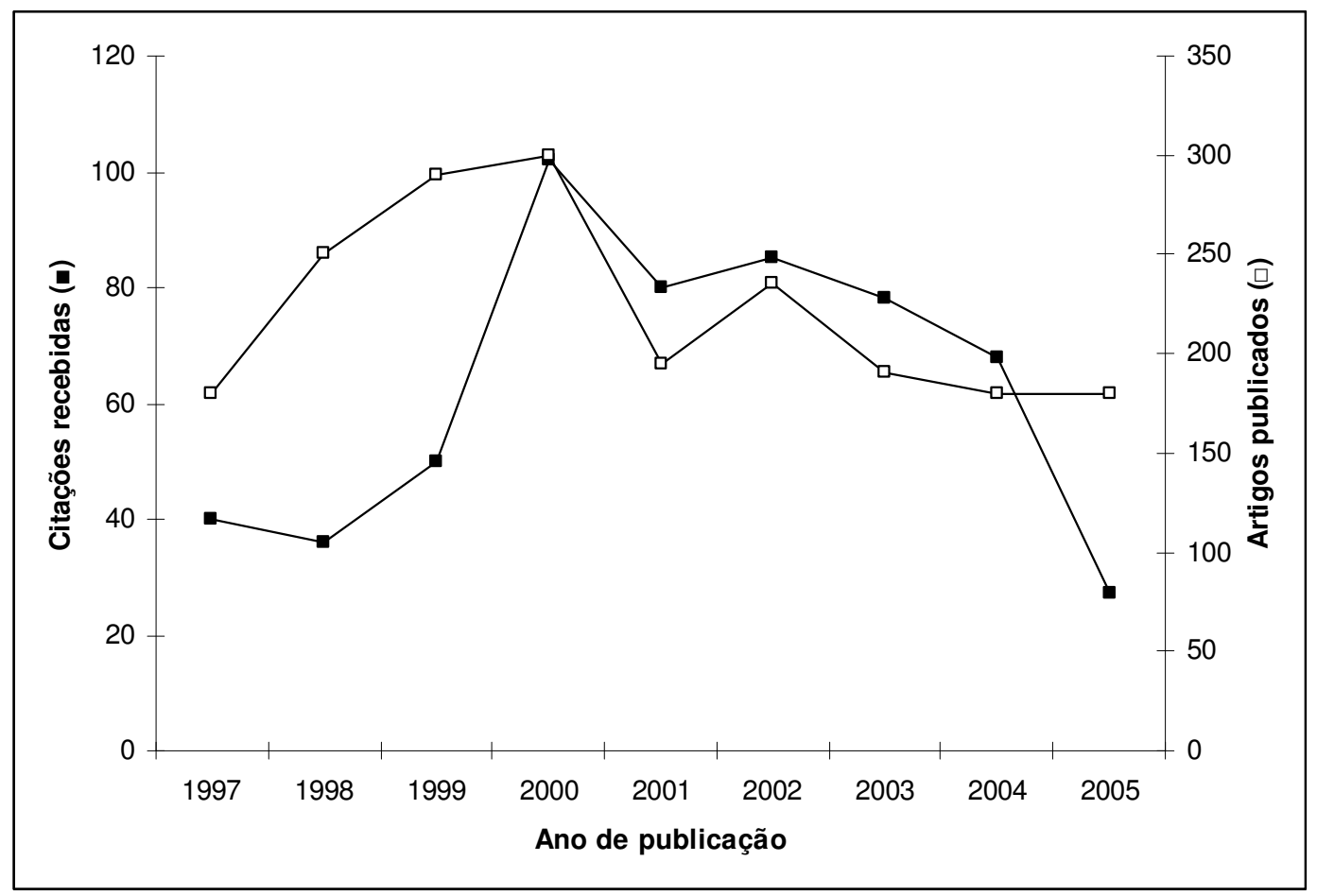

Figura 1 - Número de artigos publicados anualmente pela Pesquisa Agropecuária Brasileira (PAB) entre 1997 e 2005, e total de citações recebidas em 2006 de revistas integrantes da biblioteca SciELO, por artigos publicados pela PAB nos respectivos anos. Dados extraídos de SCIELO (2007).

FONTE - Figura elaborada a partir dos Dados extraídos de SciELO (2007).

\section{Discussão final}

Apesar de o correio eletrônico e outras tecnologias permitirem a troca de informações sobre resultados de trabalhos entre os pesquisadores, formas mais tradicionais como encontros, congressos e seminários, continuam sendo considerados espaços privilegiados para essa atividade informacional (ANDALÉCIO; MARTELETO, 2006). Da mesma forma, na chamada sociedade da informação - em que muitas vezes a informação é produzida, montada, embalada, enviada e vendida de forma autônoma e descontextualizada, e na qual são experimentadas transformações significativas no campo da comunicação científica - o periódico científico se sustenta como legitimador da autoria das descobertas científicas e da qualidade da ciência. Independentemente dos formatos assumidos - impresso ou eletrônico (em suportes variados) - o periódico científico configura-se como o meio mais eficiente e desejado de divulgação de resultados de pesquisa e de visibilidade do autor, ao disseminar informações científicas na forma de artigos, comunicações, recensões ou relatos de experiência.

Mueller (1999) afirma que os periódicos científicos publicados pelos países que não estão na fronteira do desenvolvimento da ciência e não têm o inglês como língua nacional, não têm o conceito de um periódico de 
primeira linha: em geral, não conseguem entrar no círculo de periódicos regularmente analisados pelos prestigiosos índices de citação. Como solução para esta questão, a pesquisadora destaca o periódico eletrônico, de acesso gratuito e formato não tradicional, especialmente pela melhor visibilidade proporcionada e recuperação pelos sistemas de busca.

Um estudo recente do ISI registrou que revistas tradicionais e revistas que promovem acesso livre à informação têm fatores de impacto de citação similares (PRINGLE, 2004). Entretanto, Lawrence (2001) registrou que artigos online são citados 4,5 vezes mais que artigos offline, quando considerados os artigos publicados no mesmo ano, no período de 1990 a 2000. Analisando o impacto de artigos de acesso livre contra o de artigos de acesso restrito, nos mesmos periódicos e fascículos, Harnad e Brody (2004) concluiram que o acesso livre aumenta drasticamente o número de usuários potenciais de qualquer artigo, e promove maior uso e impacto.

Considerando esse contexto em que tomou lugar a inserção da revista $\mathrm{PAB}$ na mídia eletrônica, o impacto do modelo híbrido de publicação tem sido positivo sobre a revista, uma vez que os acessos aos artigos eletrônicos multiplicaram-se em mais de 50 vezes nos últimos 6 anos, apesar da revista ter mantido o mesmo número de artigos publicados por mês, aproximadamente. Os empecilhos que geralmente se atribuem ao periódico eletrônico - como a questão da proteção ao direito autoral, por causa da reprodução ilimitada, as questões da legitimidade e da qualidade da informação, as preocupações com questões de segurança e a dificuldade crescente de se obter visibilidade, devido à grande quantidade de informações e sites disponíveis - não têm impedido o crescimento da PAB no sentido de maior visibilidade.

Como visão de futuro, o Corpo Editorial da revista PAB e a Embrapa, enquanto instituição editora, buscam a manutenção da periodicidade da revista e ainda a melhoria da qualidade científica; e preocupam-se em buscar maior divulgação e distribuição nos âmbitos nacional e internacional, garantindo a presença da revista em bibliotecas especializadas do Brasil e do mundo, além de otimizar o aproveitamento da mídia eletrônica para o crescimento dos fatores de impacto da revista.

\section{Referências}

ANDALÉCIO, A. M. L.; MARTELETO, R. M. A comunicação científica e as tecnologias da informação e comunicação. In: CONFERÊCIA IBEROAMERICANA DE PUBLICAÇÕES ELETRÔNICAS NO CONTEXTO DA COMUNICAÇÃO CIENTÍFICA, 1, 2006. Brasília. Anais... Brasília: Universidade de Brasília, 2006. Disponível em: $<$ http://portal.cid.unb.br/CIPECCbr/viewpaper.php?id=30>. Acesso em: 05 mar. 2007.

BETHESDA STATEMENT ON OPEN ACCESS PUBLISHING. Released - June 20, 2003. Disponível em: 
$<$ http://www.earlham.edu/ p peters/fos/bethesda.htm>. Acesso em: 3 abr. 2007.

CAMPOS, M. Conceitos atuais em bibliometria. Arquivos Brasileiros de Oftalmologia, n. 66, p. 1-22, 2003.

DARMONI, S. J. et al. Reading factor: a new bibliometric criterion for managing digital libraries. Journal of the Medical Library Association, v. 90, n.3, p. 323-327, julho 2002.

GARVEY, W. D. Communication: the essence of science facilitating information among librarians, scientists, engineers and students. Oxford: Pergamon Press, 1979.

HARNAD, S.; BRODY, T. Comparing the Impact of Open Access (OA) vs. Non-OA Articles in the Same Journals. D-Lib Magazine, v.10, n. 6, junho 2004. Disponível em: <http://dlib.org/dlib/june04/harnad/06harnad.html>. Acesso em: 09 abr. 2007.

INSTITUTO BRASILEIRO DE INFORMAÇÃO EM CIÊNCIA E TECNOLOGIA. Manifesto brasileiro de Apoio ao acesso livre à informação científica. Brasília: Ibict, 2005.2 Disponível em: $<$ http://www.ibict.br/openaccess/arquivos/manifesto.htm>. Acesso em: 3 abr. 2007.

LAWRENCE, S. Online or invisible? Nature, v. 411, n. 6837, p. 521, 2001.

MEADOWS, J. Can we really see where electronic journals are going? Library Management, Bradford, v. 18, n. 3, p. 151-154, 1997.

MUELLER, S. P. M. O círculo vicioso que prende os periódicos nacionais. DataGramaZero - Revista de Ciência da Informação, n. zero, artigo 04, dezembro $1999 . \quad$ Disponível em: <http://www.dgz.org.br/dez99/Art 04.htm>. Acesso em: 24 out. 2007.

MUELLER, S. P. M.; PASSOS, E.J.L. (Org.). Comunicação científica. Brasília: DCI/UnB, 2000.

PRINGLE, J. Do open access journals have impact? Nature (Web Focus). Disponível em: $<$ http://www.nature.com/nature/focus/accessdebate/19.html>. Acesso em: 9 abr. 2007.

SCIENTIFIC ELECTRONIC LIBRARY ONLINE - SCIELO. Disponível em: $<$ www.scielo.org>. Acesso em: 26 mar. 2007.

SUBER, P. Timeline of the Open Access Movement. Disponível em: <http://www.earlham.edu/ peters/fos/timeline.htm>. Acesso em: 3 abr. 2007.

TENOPIR, C.; KING, D. W. A publicação de revistas eletrônicas: economia da produção, distribuição e uso. Ciência da Informação, Brasília, DF, v. 27, n. 2, p. 176-182, maio/ago. 1998. 